Mitteilungen der Österreichischen Geographischen Gesellschaft,

157. Jg. (Jahresband), Wien 2015, S. 341-360

\title{
Berichte und Kleine Mitteilungen
}

\section{„ÖTSCHER:REICH. DIE ALPEN UND WIR ${ }^{66}$ \\ Eine Landesausstellung als Initiator einer eigenständigen und nachhaltigen Regionalentwicklung}

\author{
Werner B̈̈TZING, Erlangen/Bamberg* \\ mit 1 Kasten und 2 Tab. im Text
}

\section{INHALT}

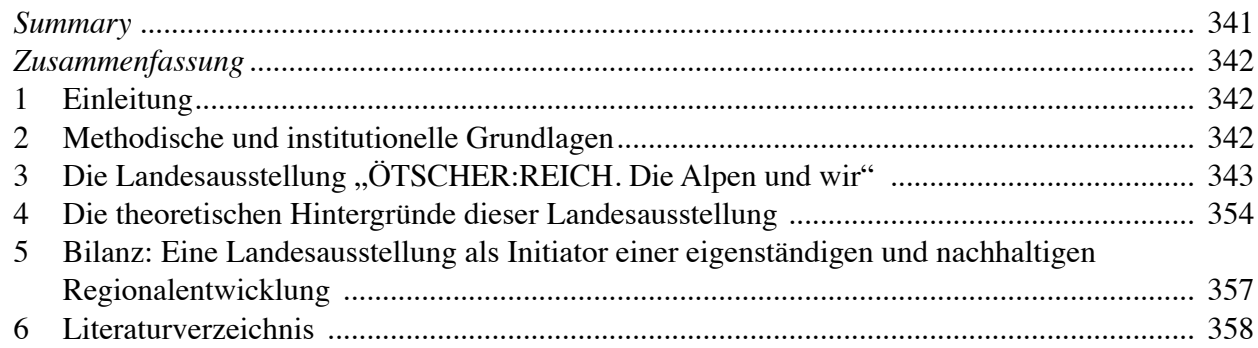

\section{Summary}

"Ötscher:Reich. The Alps and we". A provincial exhibition as initiator of an independent and sustainable regional development

In this article a new concept of an exhibition on Lower Austria [Niederösterreich] is presented intending to promote the entire exhibition region sustainably. To achieve this, the leading idea, the most important steps of implementation, experiences so far collected and theoretical backgrounds are explained. The article is to stimulate a transfer of this new concept also to other regions.

\footnotetext{
* Prof. em. Dr. Werner Bätzing, Institut für Geographie, Universität Erlangen-Nürnberg und Archiv für integrative Alpenforschung, Geyerswörthstr. 12, D-96047 Bamberg; E-Mail: werner.baetzing@web.de, http:// www.geographie.nat.uni-erlangen.de/personen/wbaetzing
} 


\section{Zusammenfassung}

In diesem Artikel wird ein neues Ausstellungskonzept aus Niederösterreich vorgestellt, das das Ziel verfolgt, die gesamte Ausstellungsregion nachhaltig aufzuwerten. Dazu werden die Leitidee, die wichtigsten Umsetzungsschritte, die bisherigen Erfahrungen sowie die theoretischen Hintergründe dargelegt.Dieser Artikel möchte dazu anregen, dass dieses neue Konzept auch in anderen Regionen angewandt wird.

\section{Einleitung}

Die alle zwei Jahre durchgeführte Niederösterreichische Landesausstellung fand im Jahr 2015 in der Ötscherregion im Südwesten des Landes statt und thematisierte unter dem Titel „ÖTSCHER:REICH. Die Alpen und wir“ das Verhältnis der Regionsbewohner und -besucher zu den Alpen, wobei die Ötscherregion als Teil der Nördlichen Kalkalpen explizit in den Kontext des gesamten Alpenraumes gestellt wurde.

Die besondere Bedeutung dieser Ausstellung liegt darin, dass sie diese periphere und strukturschwache Alpenregion aufzuwerten versucht, indem sie erstens über die beiden Ausstellungsstandorte hinaus die gesamte Ötscherregion einbezieht und zweitens die betroffene Bevölkerung von Anfang an an der Umsetzung aktiv beteiligt, wobei die Grundsätze der ,eigenständigen Regionalentwicklung" (vgl. Abschnitt 4.1) die Leitlinien der Aufwertung darstellen.

Dies ist aus einem doppelten Grund bemerkenswert: Erstens scheint eine zeitlich limitierte und auf zwei Standorte konzentrierte Ausstellung mit der Leitidee der zeitlich unbegrenzten und flächenhaft ausgerichteten eigenständigen Regionalentwicklung inhaltlich nicht vereinbar zu sein, und zweitens dominieren in Europa derzeit zumeist neoliberale Raumvorstellungen (die großen Metropolen als Motoren der Volkswirtschaften, wobei man den Peripherien nur dann eine Zukunft zubilligt, wenn sie mit schnellen Verkehrsinfrastrukturen eng mit dem nächsten Zentrum verbunden werden), sodass die Aufwertung einer Region mittels der Idee der eigenständigen Regionalentwicklung äußerst ungewöhnlich ist.

Aufgabe und Ziel dieses Artikels ist es, dieses außergewöhnliche Modell und die bislang damit gemachten Erfahrungen vorzustellen, seine innovative Bedeutung für eine nachhaltige Regionalentwicklung aufzuzeigen und dadurch zur Nachahmung in anderen Regionen der Alpen und Europas anzuregen.

\section{Methodische und institutionelle Grundlagen}

Die Niederösterreichischen Landesausstellungen finden alle zwei Jahre jeweils in einem anderen Viertel Niederösterreichs - Wald-, Wein-, Most- und Industrieviertel - statt. Mein erster Kontakt entstand im Rahmen der 5. Mostviertler Nachhaltigkeitskonferenz am 3. und 4. September 2012 im Pielachtal/Ötscherregion, wo die Leitideen für die Landesausstellung 2015 vorgestellt wurden. Da diese auf mich sehr überzeugend wirkten, beschloss ich, als wissenschaftlicher Berater und Experte an der Entwicklung dieser Ausstellung mitzuarbeiten.

Die gemeinsamen Arbeiten begannen im November 2012, dauerten bis zur Eröffnung der Ausstellung am 24. April 2015 und sind auch heute noch nicht beendet, wie später noch dargestellt werden wird. Die Vorbereitungskerngruppe bestand aus Kurt FARASIN (Geschäftsführer der Schallaburg Kulturbetriebsges.m.b.H., die die Landesausstellungen jeweils vorbereitet), aus den beiden Ausstellungsgestaltern Beat Gugger und Gerhard Proksch, aus Prof. i.R. Dr. Ernst BruckmülLer 
(Institut für Wirtschafts- und Sozialgeschichte der Universität Wien und Österreichische Akademie der Wissenschaften), aus mir und ab Anfang 2014 auch aus dem Geographen Hannes HoFFERT-HösL (Firma „Georaum GmbH“, Sankt Anton an der Jeßnitz). Je nach Bedarf wurden weitere Experten und Akteure aus der Region einbezogen.

Als Ergebnis entstanden die beiden Ausstellungen in Laubenbachmühle (Gemeinde Frankenfels) und in Neubruck (Gemeinden Sankt Anton an der Jeßnitz und Scheibbs), die durch einen umfangreichen Katalog (SchallabURg 2015), einen wissenschaftlichen Tagungsband (BRUCKMÜLleR 2015) und ein Wanderbuch (BätZING \& HofFert-HösL 2015) vertieft wurden. Dabei stand jeweils die Region rund um den Ötscher mit ihren naturräumlichen Voraussetzungen, ihrer Geschichte, ihrer heutigen Situation und ihren Zukunftsperspektiven im Zentrum, die jeweils in den alpenweiten Kontext gestellt wurden.

Der hier vorgelegte Artikel basiert auf meinen intensiven Vorbereitungen und auf meinen Erfahrungen mit dieser Landesausstellung. Diese Thematik wurde dann in ausführlichen Gesprächen mit Kurt FARASIN und Hannes HofFERT-HösL, denen ich wichtige Anregungen, Informationen und Hinweise verdanke, in der Zeit von Mai bis Juli 2015 weiter vertieft.

Das bedeutet, dass die hier vorgelegte Darstellung und Bilanzierung nicht von außen, also aus einer neutralen Beobachterperspektive erfolgt, sondern aus der Binnenperspektive eines am Prozess aktiv beteiligten Wissenschafters. Erst durch diese fachliche und emotionale Nähe wurde es möglich, diese besondere Ausstellungskonzeption in ihrer prinzipiellen Bedeutung wahrzunehmen und zu verstehen, die man andernfalls vielleicht übersehen hätte. Engagement und Wissenschaft schlieBen sich in meinem Verständnis nicht gegenseitig aus, sondern bedingen sich wechselseitig - nur als „Engagierte Geographie“ (BOESCH 1989) kann die Geographie als Wissenschaftsdisziplin ihre Verantwortung für die Welt wahrnehmen, in der sie forscht, lehrt und lebt.

\section{Die Landesausstellung „ÖTSCHER:REICH. Die Alpen und wir“}

\subsection{Vorgeschichte}

Die 1907 eröffnete Mariazellerbahn (Schmalspurbahn, bereits 1911 elektrifiziert) führt von Sankt Pölten durch das Pielachtal und am Ötscher vorbei nach Mariazell in der Steiermark. In der zweiten Hälfte der 1980er Jahre wurde sie wie viele andere Nebenbahnen zuerst von den Österreichischen Bundesbahnen (ÖBB) entwertet (Einstellung des Güterverkehrs), und später wurde sogar ihre Stilllegung diskutiert. Im Januar 2010 wurde ihre Übernahme durch das Land Niederösterreich vereinbart, und seit dem 12. Dezember 2010 wird die Mariazellerbahn von der Niederösterreichischen Verkehrsorganisationsgesellschaft (NÖVOG) betrieben.

Das Land Niederösterreich investierte bis zum Jahr 2014 insgesamt 117 Mio. Euro, um aus einer lang vernachlässigten Nebenbahn eine attraktive Regionalbahn zu machen. Für neue Fahrzeuge neuer Markenname „Die Himmelstreppe“ - wurden 65 Mio. Euro, für Verbesserungen an Gleisen, Stromversorgung und Zugsicherung 32 Mio. Euro und für ein neues Betriebszentrum 20 Mio. Euro ausgegeben (www.noevog.at). Besonders wichtig war dabei der Neubau des Betriebszentrums mit seinen 25 Arbeitsplätzen, das vom Zentrum Niederösterreichs, dem „Alpenbahnhof“ in Sankt Pölten, in die Peripherie, ins Pielachtal (Bezirk Sankt Pölten Land) verlegt wurde. Dabei entschieden sich die Gemeinden dieses Tales in einem demokratischen Prozess für den Standort Laubenbachmühle in der Gemeinde Frankenfels (Beginn der Bergstrecke der Mariazellerbahn). Hier wurde 2012/13 eine große Bahnremise errichtet, die dann im Jahr 2015 während der Landesausstellung als Ausstellungsort genutzt wurde. 
Mit der Aufwertung der Mariazellerbahn durch das Land Niederösterreich lag es auf der Hand, dass bei der nächsten Landesausstellung im Mostviertel diese Bahn eine herausgehobene Rolle spielen würde. Deshalb war es kein Zufall, dass sich das Pielachtal als erster Kandidat für die Durchführung der Landesausstellung 2015 im Mostviertel bewarb. Dies löste in der Region zahlreiche Aktivitäten aus, weil auch andere Gemeinden von der Landesausstellung profitieren wollten, was zur Bewerbung der Gemeinde Annaberg (Bezirk Lilienfeld, mit einem Bahnhof an der Mariazellerbahn) und der Gemeinden der „Eisenstraße“ (Bezirk Scheibbs, westlicher Teil der Ötscherregion) führte.

Angesichts dieser Interessenkonstellation wurde beschlossen, die Landesausstellung 2015 an zwei Standorten - Laubenbachmühle/Pielachtal und Neubruck/Eisenstraße - durchzuführen und zur Stärkung der Mariazellerbahn am Bahnhof in Wienerbruck (Gemeinde Annaberg) ein Besucherzentrum für den Naturpark Ötscher-Tormäuer zu errichten (als dritter Standort, allerdings ohne Ausstellung) sowie die Gemeinde Mariazell (Steiermark) als Endpunkt der Bahnstrecke in die Landesausstellung miteinzubeziehen. Damit war zugleich klar, dass der Gipfel des Ötschers, 1.893 m, und der Naturpark Ötscher-Tormäuer (fünf Gemeinden) den Kern dieser Landesausstellung darstellten, und dass damit „,die Alpen“ den Themenschwerpunkt bilden müssten, um die unterschiedlichen Teilgebiete sinnvoll miteinander vernetzen zu können.

Diese räumliche und thematische Leitidee wurde übrigens nicht zentralistisch auf der Schallaburg oder in Sankt Pölten festgelegt, sondern partizipativ unter Einbezug aller betroffenen Gemeinden erarbeitet, wofür es vieler intensiver Diskussionen und dreier Workshops bedurfte. Am 5. Juni 2012 wurde das Ergebnis dann von Landeshauptmann Dr. Erwin Pröll im Landhaus offiziell vorgestellt, und damit stand der Ausgangspunkt für die anschließenden Vorbereitungsarbeiten und Umsetzungsschritte fest.

\subsection{Konkretisierung der Leitidee}

Die inhaltliche Vorbereitung begann damit, dass sich die Vorbereitungskerngruppe mit der Region um den Ötscher herum vertraut machte. Naturraum, Geschichte und heutige Situation wurden daraufhin befragt, ob hier eher regionsspezifische oder eher alpentypische Verhältnisse zu finden seien, ob diese Region Besonderheiten im alpenweiten Rahmen besitze und welches die zentralen Botschaften der Landesausstellung sein könnten. Als Ergebnis lässt sich überblicksmäßig festhalten (Details siehe Schallaburg 2015):

Naturraum: Die Niederösterreichischen Kalkalpen besitzen in der Ötscherregion aufgrund des kleinräumigen Wechsels von weichen und harten Gesteinen den Charakter einer ,geologischen Musterlandschaft“ (Krebs 1928, S. 335). Die Gipfel sind hier zwar nicht mehr besonders hoch, aber dafür gibt es ausgeprägte Schluchten (Vordere und Hintere Tormäuer, Ötschergraben), und wir finden hier mit dem „Wildnisgebiet Dürrenstein“ den größten Urwald Mitteleuropas und der Alpen.

Geschichte: Das Pielachtal in der Nähe des Alpenrandes ist ein ehemaliges Herrschaftsgebiet von weltlichen Grundherren, während das gesamte übrige Gebiet eine lange klösterliche Herrschaft erlebte (Klöster Gaming, Lilienfeld, Sankt Lambrecht). Trotz dieser unterschiedlichen Herrschaftsstrukturen ist die gesamte Region bis heute stark kleinbäuerlich geprägt. Seit dem ausgehenden Mittelalter gehört sie zu den „Eisenwurzen“ (stark arbeitsteilig geprägte kleingewerbliche Verarbeitung des vom steirischen Erzberg stammenden Roheisens), und dies führt zu einer starken gewerblichen Tradition und einer intensiven Waldnutzung. Im Ostteil gibt es besonders viele Pilgerwege (mit Ansätzen einer barocken „Sakrallandschaft“ entlang der Via Sacra) und in Mariazell selbst ein florierendes Andenken- und Devotionaliengewerbe. Im 18. Jh. führte der sehr stark wachsende Brennholzbedarf der Stadt Wien zu sehr großen kurzfristigen und flächenhaften Rodungen der letzten, schlecht erreichbaren Wälder, für die evangelische Holzfäller aus dem Salzkammergut angeworben wurden 
und die später in Mitterbach die erste evangelische Pfarrei Niederösterreichs gründeten. Mit der Säkularisierung endete die Klosterherrschaft, und ab 1870 erwarben großbürgerliche Unternehmer aus Wien große Teile der früheren Klosterwälder zu jagdlichen Zwecken, was mit deutlichen Waldvergrößerungen - u.a. durch das sogenannte „Bauernlegen“- verbunden war. Deshalb gehören große Teile der Ötscherregion heute zu den am stärksten bewaldeten Gebieten der gesamten Alpen. Weil im Gebiet der Eisenwurzen der Strukturwandel hin zur Industrieproduktion nicht wirklich gelang, ging die Bedeutung des II. Wirtschaftssektors hier seit dem Ende des 19. Jhs. deutlich zurück. Der Tourismus hielt zwar mit dem Bau der Mariazellerbahn - einer typischen touristischen Alpenbahn - in der Ötscherregion Einzug, aber er verblieb bis heute in kleinen, wenig konkurrenzfähigen Strukturen.

Heutige Situation: Die Ötscherregion ist eine durch Auspendler geprägte ländliche Region ohne ein größeres städtisches Zentrum, die abseits der großen Verkehrsströme, aber nicht völlig peripher liegt. Stattdessen ist die örtliche Umwelt- und Lebensqualität hoch, und Schutzgebiete wie der Naturpark Ötscher-Tormäuer und das Wildnisgebiet Dürrenstein spielen eine relevante Rolle. Der Bezug der Bewohner zu ihrer Gemeinde ist in der Regel stark ausgeprägt, was sich in einem lebendigen Vereinsleben und in zahllosen kulturellen Aktivitäten und Festen niederschlägt. Von herausragender Bedeutung ist auch heute noch der „Gnadenort Mariazell“, der größte Wallfahrtsort der gesamten Alpen und einer der bedeutendsten Wallfahrtsorte ganz Europas, dessen Pilgerwege auch durch die Ötscherregion verlaufen.

Zusammenfassung: Diese Ergebnisse aus den Bereichen Naturraum, Geschichte und heutige Situation bildeten die Grundlage für die Gestaltung der Ausstellungen an den zwei Ausstellungsorten und für die Ausstellungspublikationen. Ziel war es, keine austauschbaren Inhalte und Events zu präsentieren, sondern die Region selbst in den Mittelpunkt zu stellen, ihre verborgenen und leicht zu übersehenden Qualitäten und Potenziale herauszustellen und erlebbar zu machen, ihre Probleme und ihre zukünftige Entwicklung zu thematisieren und durch den gezielten Vergleich mit dem gesamten Alpenraum die Wahrnehmung sowohl der regionsspezifischen wie der alpentypischen Situationen und Entwicklungen zu schärfen.

Regionsabgrenzung: Erst vor diesem inhaltlichen Hintergrund war es der Vorbereitungsgruppe dann möglich, die „Ötscherregion“ im Detail auf Gemeindeebene abzugrenzen (vgl. Tab. 1). Sie besteht aus 17 Gemeinden ${ }^{1}$, die in vier unterschiedlichen Politischen Bezirken und in zwei Bundesländern liegen. Diese unübliche Regionsabgrenzung, die quer zu allen politischen Strukturen steht, erklärt sich aus der Leitidee der Landesausstellung, die Gemeinden rund um den Ötscher zusammenzufassen, um sie gemeinsam zu stärken. Alle Gemeinden der Ötscherregion liegen in den Alpen und besitzen eine periphere Lage, was ihre Entwicklung jeweils erschwert, sodass es sich anbietet, sie gezielt miteinander zu vernetzen, um ihre Entwicklungspotenziale zu stärken.

Eine solche Raumgliederung entspricht wichtigen Erfahrungen, die bei der Aufwertung von ländlichen Räumen gemacht wurden: Wenn Räume nach dem Zentrale-Orte-Prinzip abgegrenzt werden - wie es häufig geschieht -, dann liegt im Mittelpunkt jeder politischen Raumeinheit ein Zentraler Ort (Ober-, Mittel- oder Unterzentrum), der seine Umgebung mit zentralen Dienstleistungen versorgt. Die ländlichen Räume liegen dann jeweils am Rand oder in der Peripherie dieser Raumeinheiten, und sie werden - als Räume zwischen den Zentren - durch diese Grenzen zerschnitten und auf mehrere dieser Raumeinheiten aufgeteilt. Bei einer solchen Raumgliederung wird der ländliche Raum also zerstückelt und überall zum Grenzraum gemacht. Weil diese Grenzen im Alltag große Bedeutung besitzen, erschweren oder verhindern sie die Kommunikation, die gemeinsame Nutzung von regionalen Ressourcen und deren gemeinsame Vermarktung. Deshalb schwächt eine

1) Zum 1. Januar 2015 wurden die Gemeinden Mariazell, Halltal, Sankt Sebastian und Gußwerk zur neuen Großgemeinde Mariazell zusammengelegt. Da bei Statistik Austria hierfür noch keine Daten erhältlich sind, werden hier weiterhin die Daten der alten Kleingemeinden verwendet. 
solche Raumgliederung, die sich an den Zentralen Orten orientiert, den ländlichen Raum und verstärkt dessen Charakter als Peripherie noch zusätzlich. ${ }^{2)}$

\begin{tabular}{|c|c|c|c|}
\hline & Fläche $\left(\mathbf{k m}^{2}\right)$ & Höhe (NN) & $\begin{array}{l}\text { Einwohner } 2011 \\
\text { (Volkszählung) }\end{array}$ \\
\hline \multicolumn{4}{|l|}{314 Lilienfeld } \\
\hline 01 Annaberg & 63,52 & $976 \mathrm{~m}$ & 562 \\
\hline \multirow[t]{2}{*}{08 Mitterbach am Erlaufsee } & 67,32 & $791 \mathrm{~m}$ & 548 \\
\hline & 130,84 & & \\
\hline \multicolumn{4}{|l|}{319 St. Pölten Land } \\
\hline 06 Frankenfels & 56,16 & $465 \mathrm{~m}$ & 2.040 \\
\hline 09 Hofstetten-Grünau & 36,00 & $317 \mathrm{~m}$ & 2.570 \\
\hline 18 Kirchberg a.d. Pielach & 63,41 & $372 \mathrm{~m}$ & 3.116 \\
\hline 20 Loich & 24,58 & $442 \mathrm{~m}$ & 607 \\
\hline 35 Rabenstein a. d. Pielach & 36,22 & $344 \mathrm{~m}$ & 2.403 \\
\hline \multirow[t]{2}{*}{39 Schwarzenbach/Pielach } & 45,37 & $510 \mathrm{~m}$ & 392 \\
\hline & 261,74 & & \\
\hline \multicolumn{4}{|l|}{320 Scheibbs } \\
\hline 01 Gaming & 243,87 & $430 \mathrm{~m}$ & 3.244 \\
\hline 02 Göstling a. d. Ybbs & 143,66 & $532 \mathrm{~m}$ & 2.085 \\
\hline 05 Lunz am See & 101,66 & $601 \mathrm{~m}$ & 1.842 \\
\hline 07 Puchenstuben & 41,29 & $869 \mathrm{~m}$ & 319 \\
\hline 11 St. Anton a. d. Jeßnitz & 69,70 & $390 \mathrm{~m}$ & 1.229 \\
\hline \multirow[t]{2}{*}{13 Scheibbs } & 45,96 & $341 \mathrm{~m}$ & 4.199 \\
\hline & 646,14 & & \\
\hline \multicolumn{4}{|l|}{621 Bruck-Mürzzuschlag } \\
\hline 11 Halltal & 74,48 & $810 \mathrm{~m}$ & 336 \\
\hline 17 Mariazell & 6,44 & $868 \mathrm{~m}$ & 1.492 \\
\hline \multirow[t]{2}{*}{30 St. Sebastian } & 47,40 & $820 \mathrm{~m}$ & 1.005 \\
\hline & 128,32 & & \\
\hline Ötscherregion & $1.167,04$ & & 27.989 \\
\hline
\end{tabular}

Quelle: Statistik Austria/Gemeindedatenbank, BÄTZING

Tab. 1: Die Gemeinden der Ötscherregion (gegliedert nach Politischen Bezirken)

2) Siehe dazu Bätzing 2015a und Sondershaus 2008. Auf der Maßstabsebene der europäischen Makroregionen wird dies ebenfalls diskutiert: Können die Alpen als Peripherie - also ohne die großen Zentren wie Wien, München, Mailand [Milano] usw. - eine eigene Makroregion bilden oder ist eine solche Makroregion nur dann denkbar, wenn dabei die großen Zentren am Rande der Alpen in die Alpen miteinbezogen werden? 


\begin{tabular}{|c|c|c|}
\hline \multicolumn{3}{|l|}{314 Lilienfeld } \\
\hline 01 Annaberg & $\mathrm{A}$ & $\mathrm{B}$ \\
\hline 04 Hohenberg & & $\mathrm{B}$ \\
\hline 07 Lilienfeld & & $\mathrm{B}$ \\
\hline 08 Mitterbach a. E. & $\mathrm{A}$ & $\mathrm{B}$ \\
\hline 11 St. Aegyd & & $\mathrm{B}$ \\
\hline 14 Türnitz & & $\mathrm{B}$ \\
\hline \multicolumn{3}{|l|}{319 St. Pölten Land } \\
\hline 06 Frankenfels & $\mathrm{A}$ & $\mathrm{B}$ \\
\hline 09 Hofstetten-Grünau & $\mathrm{A}$ & $\mathrm{B}$ \\
\hline 18 Kirchberg a. d. P. & $\mathrm{A}$ & $\mathrm{B}$ \\
\hline 20 Loich & $\mathrm{A}$ & $\mathrm{B}$ \\
\hline $29 *$ Ober-Grafendorf & & $\mathrm{B}$ \\
\hline 35 Rabenstein a. d. P. & A & $\mathrm{B}$ \\
\hline 39 Schwarzenbach/P. & $\mathrm{A}$ & $\mathrm{B}$ \\
\hline $45 *$ Weinburg & & $\mathrm{B}$ \\
\hline \multicolumn{3}{|l|}{320 Scheibbs } \\
\hline 01 Gaming & $\mathrm{A}$ & $\mathrm{B}$ \\
\hline 02 Göstling a. d. Y. & A & $\mathrm{B}$ \\
\hline 03 Gresten & & $\mathrm{B}$ \\
\hline 04 Gresten-Land & & $\mathrm{B}$ \\
\hline 05 Lunz a. S. & $\mathrm{A}$ & $\mathrm{B}$ \\
\hline $06 *$ Oberndorf & & $\mathrm{B}$ \\
\hline 07 Puchenstuben & $\mathrm{A}$ & $\mathrm{B}$ \\
\hline $08 *$ Purgstall & & $\mathrm{B}$ \\
\hline 09 Randegg & & $\mathrm{B}$ \\
\hline 10 Reinsberg & & $\mathrm{B}$ \\
\hline 11 St. Anton a. d. J. & $\mathrm{A}$ & $\mathrm{B}$ \\
\hline 12 St. Georgen a. d. L. & & $\mathrm{B}$ \\
\hline 13 Scheibbs & $\mathrm{A}$ & $\mathrm{B}$ \\
\hline 14 Steinakirchen & & $\mathrm{B}$ \\
\hline $16 *$ Wieselburg & & $\mathrm{B}$ \\
\hline $17 *$ Wieselburg-Land & & $\mathrm{B}$ \\
\hline $18 *$ Wolfpassing & & $\mathrm{B}$ \\
\hline \multicolumn{3}{|c|}{621 Bruck-Mürzzuschlag } \\
\hline 11 Halltal & $\mathrm{A}$ & \\
\hline 17 Mariazell & $\mathrm{A}$ & \\
\hline 30 St. Sebastian & A & \\
\hline
\end{tabular}

A Gemeinden der Ötscherregion (17 Gemeinden)

B Gemeinden der „Landesausstellungsregion“ (31 Gemeinden)

* Gemeinde außerhalb des Geltungsbereichs der Alpenkonvention (www.alpconv.org/en/organization/parties/Documents/Administrative_Units_AC.pdf)

Tab. 2: Die Gemeinden der Ötscherregion und der „Landesausstellungsregion“ (gegliedert nach Politischen Bezirken) 
Es war von großer Wichtigkeit, dass bei der Gebietsabgrenzung der Ötscherregion diese Erfahrungen bewusst reflektiert wurden, sodass eine Raumgliederung gewählt wurde, die der Aufgabenstellung entsprach, nämlich eine Region zu bilden, die kein großes Zentrum besitzt und die zahlreiche periphere Gemeinden grenzüberschreitend zusammenfasst.

Parallel zu dieser wissenschaftlich-systematischen Regionsabgrenzung, die für die Erarbeitung der Ausstellungsinhalte eine wichtige Rolle spielte, wurde auf der politischen Ebene von den betroffenen Regionen und Gemeinden in Abstimmung mit den Verantwortlichen der Landesausstellung eine sogenannte „Landesausstellungsregion“ festgelegt, deren Grenzen für die Teilnahme an verschiedenen Förderprogrammen der Landesausstellung wichtig waren. Diese „Landesausstellungsregion" geht an drei Stellen deutlich über die Ötscherregion hinaus und umfasst 17 zusätzliche Gemeinden (vgl. Tab. 2). Dadurch vergrößert sich ihre Fläche gegenüber der Ötscherregion um 71\%, und die Zahl der Einwohner steigt von knapp 28.000 auf knapp 69.000 Einwohner im Jahr 2011 an.

Diese Gebietserweiterung ist nicht ganz unproblematisch, weil dadurch sieben Gemeinden außerhalb des Alpenraumes (außerhalb des Geltungsbereichs der Alpenkonvention) einbezogen werden, die aufgrund ihrer günstigen Lage und Erreichbarkeit im Alpenvorland eine ziemlich positive Wirtschafts- und Bevölkerungsentwicklung verzeichnen, sodass sie eine deutlich andere Ausgangssituation als die Gemeinden der Ötscherregion besitzen. Damit wird die Leitidee der Regionsabgrenzung etwas verwässert. Der folgende Text bezieht sich deshalb auf die aus 17 Alpengemeinden bestehende Ötscherregion.

\subsection{Eigenschaften und Struktur der Ötscherregion}

Die 17 Gemeinden der Ötscherregion umfassen eine Fläche von $1.167 \mathrm{~km}^{2}$ mit 27.989 Einwohnern im Jahr 2011 (Volkszählungsdaten), was mit 24 Einwohnern/ $\mathrm{km}^{2}$ eine sehr niedrige Einwohnerdichte bedeutet (Alpen insgesamt: $79 \mathrm{E} / \mathrm{km}^{2}$, Österreich: $102 \mathrm{E} / \mathrm{km}^{2}$ ). Der Waldanteil der Region beträgt $75 \%$, und er liegt bei acht Gemeinden sogar bei über $80 \%$ der Gemeindefläche (siehe dazu BorSDORF 2005, S. 22). Der Naturpark Ötscher-Tormäuer umfasst $170 \mathrm{~km}^{2}$, das Wildnisgebiet Dürrenstein $35 \mathrm{~km}^{2}$, sodass die geschützten Flächen 18\% der Regionsfläche ausmachen.

Diese ländliche Region besitzt kein einziges größeres Zentrum - die mit Abstand größte Gemeinde ist die Stadt Scheibbs mit 4.199 Einwohnern - und eine negative Pendlerbilanz, deren negativer Saldo zwischen 2001 und 2011 leicht zunimmt. Von den 17 Gemeinden sind 14 Auspendlergemeinden, die Gemeinde Göstling ist eine tertiär geprägte Gemeinde, und nur Scheibbs und Mariazell stellen Einpendlergemeinden dar. ${ }^{3)}$ Die Erwerbstätigen am Arbeitsort gingen zwischen 2001 und 2011 leicht zurück (-3,5\%), aber hierbei fällt auf, dass es in der Region noch 1.366 landund forstwirtschaftliche Betriebe gibt, was ein relevantes endogenes Potenzial darstellt. Dies schlägt sich in einem sehr hohen Anteil der Erwerbstätigen am Arbeitsort im I. Wirtschaftssektor nieder, der mit 15\% im Jahr 2011 dreimal so hoch wie der österreichische Durchschnitt (5\%) ist. Die lange gewerbliche Tradition macht sich im II. Wirtschaftssektor nicht mehr bemerkbar, der mit $26 \%$ genau dem Wert für Österreich entspricht. Und der III. Wirtschaftssektor ist in der Ötscherregion mit nur $59 \%$ signifikant schwächer als in Österreich (69\%) ausgeprägt. Diese wirtschaftlichen Strukturdaten werden üblicherweise als Ausdruck für eine schwierige wirtschaftliche Lage interpretiert.

Betrachtet man die Bevölkerungsentwicklung der Ötscherregion und stellt sie in den alpenweiten Kontext, so gibt es ein überraschendes Ergebnis: Zwischen 1869 und 2011 wächst die Einwohnerzahl der Region nur um 14,5\%, was als Stagnation zu bewerten ist, während der gesamte Alpenraum in dieser Zeit um 94\% wächst, also seine Einwohnerzahl nahezu verdoppelt. Allerdings sind die Alpen

3) Die neue Großgemeinde Mariazell dürfte eine ausgeglichene Pendlerbilanz besitzen und damit als Einpendlergemeinde wegfallen. 
dabei durch starke interne Gegensätze geprägt: $55 \%$ aller Gemeinden verzeichnen ein oft starkes Wachstum, 36\% einen oft starken Rückgang, und nur 9\% aller Gemeinden eine Stagnation (von -9\% bis $+10 \%$ ). Das bedeutet, dass die Alpen fast überall durch gegenläufige Prozesse bestimmt werden, die in letzter Konsequenz zu Verstädterung oder Entsiedlung führen (vgl. BäTzING 2015a, Kap. IV, 5).

Die Ötscherregion entzieht sich dieser alpentypischen Entwicklung, indem sie zur Minderheit der Gemeinden zählt, die durch eine Bevölkerungsstagnation geprägt ist. Und die Karte „Bevölkerungsveränderung in den Gemeinden des Alpenraumes 1871 bis 2011“ (BätZING 2015a, S. 320-321) lässt gut erkennen, dass sich in der Ötscherregion die größte Konzentration von Stagnationsgemeinden im gesamten Alpenraum findet.

Was ist die Ursache für diese außergewöhnliche Entwicklung? Einerseits kann man feststellen, dass sich die Ötscherregion der modernen Entwicklung nicht verweigert hat, was zum Bevölkerungsrückgang geführt hätte (Neue Impulse in den Bereichen Gewerbe, Wasserkraft, Tourismus, Verkehr und Wissenschaft wurden aktiv aufgegriffen.), andererseits führten diese Modernisierungen aber auch nicht zu hohen Spezialisierungen und starken räumlichen Konzentrationen, was zu regionalen Monostrukturen und zur Verstädterung geführt hätte (kein richtiges Industriegebiet, keine großen Stauseen, keine großen Hotels/Skigebiete, Mariazellerbahn nur Schmalspurbahn usw.).

Ich habe diese Entwicklung als ,gebremste Modernisierung“ bezeichnet (BäTZING 2015e), die nicht auf einer bewussten und langfristigen Strategie der Entscheidungsträger beruht, sondern die aus zahllosen Einzelentscheidungen lokaler und regionaler Akteure erwachsen ist. Diese haben auf oft sehr spontan-pragmatische Weise Tradition und Moderne miteinander verbunden, oder anders ausgedrückt, es gelang ihnen immer wieder, ihr Misstrauen gegenüber allem Neuen mit einer gewissen Weltoffenheit zu vereinen. Dabei war es durchaus hilfreich, dass die Ötscherregion zwar abseits der großen Verkehrsachsen, aber auch nicht völlig peripher liegt. Auf diese Weise war es möglich, der Region eine gewisse Eigenständigkeit und strukturelle Vielfalt zu erhalten, und diese Faktoren stellen heute ihre größten Potenziale dar.

Sieht man sich jedoch die Bevölkerungsentwicklung der letzten Jahrzehnte an, so liegt der absolute Höhepunkt der Einwohnerzahl im Jahr 1971; danach stagnieren die Zahlen bis 1991, und zwischen 1991 und 2011 verliert die Ötscherregion wieder 8\% ihrer Einwohner. Das bedeutet, dass das durchaus erfolgreiche Modell der gebremsten Modernisierung ab 1991 im Rahmen neuer globaler Rahmenbedingungen (Auflösung des Kommunismus, Erstarken des Neoliberalismus, forcierte Globalisierung in Wirtschaft und Gesellschaft) nicht mehr angemessen funktioniert - die Ötscherregion bedarf jetzt gezielter Aufwertungen, um nicht zur Abwanderungsregion zu werden.

\subsection{Aufwertung der Ötscherregion durch die Landesausstellung}

Die Aufwertungen, die durch die Landesausstellung initiiert werden, lassen sich in zwei Bereiche unterteilen, in allgemeine Aufwertungen, die mit jeder Landesausstellung verbunden sind, und in spezifische Aufwertungen, die allein für die Landesausstellung in der Ötscherregion entwickelt wurden. Grundsätzlich kann man feststellen, dass mit den Niederösterreichischen Landesausstellungen relevante Geldmittel verbunden sind, und diese betrugen in den letzten Jahren oft 20-30 Mio. Euro pro Ausstellung (Fritz \& LinKe 2011).

Die allgemeinen Aufwertungen bestehen aus Investitionen in die Ausstellungsstrukturen (die zumeist von regionalen Firmen ausgeführt werden), aus Infrastrukturförderungen in den Standortgemeinden und in weiteren Gemeinden der Ausstellungsregion sowie aus einer Konzentration von Infrastrukturmaßnahmen durch das Land Niederösterreich in der gesamten Ausstellungsregion (zu den investierten Summen siehe Kasten 1). Darüber hinaus genießt die Ausstellungsregion bei zahlreichen Fördermaßnahmen im Land Niederösterreich eine gewisse Priorität, sodass bei vielen Pro- 
Kosten der Ausstellung: 6 Mio. Euro (Ausstellungsbudget, Ausstellungsarchitektur, Ausstellungsproduktion, Leihgaben, Marketing, Personal, Verwaltung)

Investitionen in die drei Ausstellungsstandorte und die Ausstellungsregion:

- Neubruck: 9,3 Mio. Euro (zuzüglich 490.000,- Euro vom Bundesdenkmalamt)

- Wienerbruck: 4,7 Mio. Euro (inkl. Schutzhaus Vorderötscher und Wegsanierungen im gesamten Naturpark Ötscher-Tormäuer)

- Restbetrag: 6 Mio. Euro (Einbauten Laubenbachmühle und regionale/kommunale Projekte)

Gesamtbetrag: 20 Mio. Euro plus 6 Mio. Euro

Quelle: NIEDERÖSTERREICHISCHE LANDESAUSSTELLUNG 2015

Kasten 1: Übersicht über die Kosten der Niederösterreichischen Landesausstellung 2015

jekten eine Bündelung von Fördermitteln (EU-, Bundes- und Landesmitteln) erreicht werden kann.

An den drei Ausstellungsstandorten gab es folgende Investitionen und Aufwertungen:

In Laubenbachmühle wurde die neue, preisgekrönte Bahnremise (innovative Verwendung von Holz) durch temporäre Einbauten zur Ausstellungshalle umgebaut.

In Neubruck wurde eine große Industriebrache aufgewertet: Das sogenannte „Töpperschloss“ der repräsentative Wohnbau eines Industriellen aus dem 19. Jh. - wurde einschließlich seiner parkartigen Umgebung und seiner Kapelle aufwändig saniert. Dieses große Gebäude wird nach der Ausstellung unter dem Namen „TöpperZwei“ in Form von Gemeinschaftsbüros („Co-Working-Space“) genutzt werden. In den ehemaligen Fabrikshallen und auf dem Firmengelände wird Platz für die Ansiedlung neuer Betriebe geschaffen, wobei die neu gegründete „Neubruck Immobilienges.m.b.H.“, an der 17 Gemeinden der Ausstellungsregion und drei Banken beteiligt sind, das Standortmarketing übernimmt.

In Wienerbruck wurde direkt am Bahnhof der Mariazellerbahn und am Beginn des Wanderwegs hinab in die Schluchten der Hinteren Tormäuer und des Ötschergrabens ein Naturparkzentrum im „Holzstadel-Stil“ errichtet (Sommerbetrieb), das Infozentrum, Restaurant, Naturparkshop, Sanitärräume, Picknickplätze und einen Veranstaltungsraum beherbergt und das als Ausgangsort für geführte Naturparkwanderungen dient. Im Rahmen der Werbung für die Landesausstellung wurde Wienerbruck wie ein dritter Standort behandelt, ,„[...] wo anstelle einer Ausstellung das neue Naturparkzentrum [...] zu einem Schnupperrundgang in den Naturpark einlädt" (Prospekt- und Internettext).

Darüber hinaus gab es in den Gemeinden der Ausstellungsregion weitere Investitionen:

- Aufwertung von Ortsbildern und Denkmalschutzmaßnahmen an öffentlichen Gebäuden und Plätzen;

- Neuanlage/Aufwertung von Wander-, Pilger-, Themen- und Erlebniswegen;

- weitere touristische Maßnahmen (Schauhöhle, Wasserprojekte, zusätzliche Eingangsgebäude für den Naturpark);

- Unterstützung von dezentralen Ausstellungen;

- Entwicklung einer eigenen Regionalproduktlinie „ÖTSCHER:REICH“ (Bier, Fisch, Honig, Marmelade);

- Ausbau der Eibeck-Alm (Gemeinde Frankenfels) und Wiedereröffnung eines Almbetriebes;

- Modernisierung der kleinen, regional ausgerichteten Schigebiete Annaberg, Gemeindealpe, Hochkar und Lackenhof;

- Straßenverbesserungen (neue Straßenbeläge, Verbreiterungen, Hangsicherungen, Kreisverkehre, Gehwege);

- Unterstützung (bei Marketing und Öffentlichkeitsarbeit) einer Vielzahl von Veranstaltungen in der Ausstellungsregion während der Ausstellungszeit. 
Weiterhin wurden durch die Landesausstellung zusätzliche Investitionen von öffentlicher und privater Seite ausgelöst:

- Neubau des Terzer-Hauses auf dem Gipfel der Gemeindealpe, 1.626 m: Investitionen von 2,4 Mio. Euro durch Bergbahnen Mitterbach und NÖVOG;

- Bau eines JUFA-Hotels in Annaberg mit 208 Betten (Fertigstellung September 2015) und in Göstling (in Planung);

- viele Ausbauten/Modernisierungen von kleinen Hotels, Pensionen, „Urlaub auf dem Bauernhof“, Restaurants, Cafés;

- Ausbauten/Modernisierungen im Bereich Direktvermarktung von Regionalprodukten.

Neben diesen allgemeinen Aufwertungen wurden für die Ötscherregion zusätzlich ganz spezifische Aufwertungen entwickelt. Die Leitidee bestand dabei darin, das Interesse der Besucher von den beiden Ausstellungsorten über die Mariazellerbahn (Das Tagesticket beinhaltete mit dem Eintritt die Fahrt mit der Bahn zwischen Laubenbachmühle und Wienerbruck.) gezielt auf die gesamte Ötscherregion zu lenken: vom Punkt (zwei bzw. drei Ausstellungsorte) über die Linie (Mariazellerbahn) zur Fläche (in die gesamte Region).

Die zentrale Voraussetzung dafür, dass dies überhaupt sinnvoll und möglich war, bestand darin, dass an den beiden Ausstellungsorten jeweils die gesamte Ötscherregion mit unterschiedlichen Schwerpunkten thematisiert und präsentiert wurde, sodass die Ausstellungen inhaltlich bereits weit über die beiden Standorte hinauswiesen. Um dies für die Besucher noch zu verstärken, wurden fünf spezifische Maßnahmen entwickelt:

1. „Regionspartner Mostviertel“: Im Jahr 2013 wurden Partnerbetriebe der Landesausstellung Gastronomie- und Beherbergungsbetriebe, Direktvermarkter, regionale Betriebe - gesucht, die sich mit den Zielen der Landesausstellung identifizierten, eine Reihe von Mindestanforderungen erfüllten und umfangreiche Weiterbildungen zum Thema Ötscherregion absolvierten. Die Nachfrage aus der Region übertraf alle Erwartungen, und zu Beginn der Landesausstellung gab es 174 offizielle „Regionspartner Mostviertel“. Diese werden in Zukunft langfristige Partner des „Mostviertel Tourismus“ bleiben.

2. „Natur- und Kulturvermittler“: Um den Besuchern der Landesausstellung die Charakteristika der Ötscherregion zu zeigen, wurden vor allem jüngere Menschen aus der Region gesucht, die nach einer gezielten Ausbildung in der Lage waren, Natur, Landschaft, Geschichte, Wirtschaft und Kultur sehr konkret (z.B. an den ÖTSCHER:REICH-Stationen) zu erklären und Gäste durch die Region zu führen. Auch hier übertraf die Nachfrage die Erwartungen, und zu Beginn der Landesausstellung gab es 83 offizielle „Naturvermittler“. Diese sollen in Zukunft für den Naturpark Ötscher-Tormäuer tätig werden. Zusätzlich wurden 33 „Kulturvermittler“ ausgebildet, die als regional verankerte Personen Besuchergruppen durch die beiden Ausstellungen führen und dabei ihre persönlichen Erfahrungen mit einbringen.

3. Naturpark Ötscher-Tormäuer: Die juristische Struktur dieses Naturparks wurde im November 2012 geändert (Der Naturparkverein, der aus fünf Mitgliedsgemeinden besteht, wurde um eine Ges.m.b.H. erweitert.), um seine Handlungsfähigkeit in Hinblick auf die Landesausstellung und die Zeit danach zu verbessern. Die größte Investition betraf den Bau des neuen Naturpark-Besucherzentrums „Ötscher-Basis“. Darüber hinaus wurde das vom Verfall bedrohte Schutzhaus Vorderötscher - ein sehr wichtiger Stützpunkt für Wanderer - vom Naturpark übernommen und saniert (Bau von Klär-, Trinkwasser- und Energieversorgungsanlagen). Es wird seitdem vom Naturpark bewirtschaftet, und es wurden sehr viele Wanderwege im gesamten Naturparkgebiet saniert und mit neuen Markierungen versehen. 
4. „ÖTSCHER:REICH-Stationen“: Auf lokale Initiative hin wurden in der gesamten Ötscherregion 15 Außenstationen ausgewiesen, an denen wichtige Themen aus Natur, Geschichte, Wirtschaft und Kultur besonders gut sichtbar und erlebbar sind. Diese spielen bei den Aktivitäten der Naturvermittler eine wichtige Rolle und sie werden in den Prospekten und auf der Internetseite der Landesausstellung für die Besucher sehr deutlich präsentiert. Diese Außenstationen besitzen von Anfang an eine eigene Trägerschaft (die jeweilige Gemeinde), damit sie nach dem Ende der Landesausstellung problemlos fortgeführt werden können.

5. Neue Wanderwege und ein neues Wanderbuch: Da es im Bereich des Ötschers bislang keinen Rundweg um diesen markanten Gipfel gab, wurde er im Kontext der Landesausstellung entwickelt. Er beginnt und endet direkt an der Bahnstation Laubenbachmühle (Der letzte Raum der dortigen Ausstellung ist der „Aufbruchsraum“, in dem „die Wanderschuhe geschnürt werden“ sollen.), besitzt am Ende jeder Tagesetappe eine Übernachtungsmöglichkeit und berührt die schönsten und wichtigsten Punkte der Ötscherregion. Parallel dazu wurde der Pielachtal-Rundweg wieder reaktiviert und seine Wegführung leicht modifiziert, sodass er jetzt ebenfalls in Laubenbachmühle beginnt und endet. Diese beiden Rundwanderwege mit 13 und 4 Tagesetappen werden zusammen mit einer Besteigung des Dürrensteins (2 Tagesetappen) im neuen Wanderbuch „Der Ötscher“ (B ÄTZING \& HOFFERT-HösL 2015) beschrieben, das für die Landesausstellung erarbeitet wurde. Es verfolgt das Ziel, die Besucher in die gesamte Region und nicht nur an wenige bekannte Punkte zu führen. Im Unterschied zu den Wanderführern der großen Verlage, die fast nur aus Wegbeschreibungen bestehen, legt dieses Wanderbuch großen Wert darauf, in die Natur, Geschichte und Kultur der durchwanderten Region einzuführen, damit man das, was man unterwegs sieht, auch angemessen versteht. Dabei übertrugen Hannes HOFFERT-HösL und ich dasjenige Wanderbuchkonzept, das ich für den piemontesischen Weitwanderweg „Grande Traversata delle Alpi/GTA“ entwickelt hatte, auf die Ötscherregion. Und da der Rotpunktverlag in Zürich nahezu der einzige Verlag im deutschsprachigen Raum ist, der großen Wert auf vertiefte Informationen zu den durchwanderten Regionen legt, erschien das Wanderbuch „Der Ötscher“ nicht in Wien oder Sankt Pölten, sondern in Zürich. Darüber hinaus unterstützte die Landesausstellung die Neu- und Wiederauflage von weiteren Ötscher-Publikationen, um das Interesse an der gesamten Ötscherregion zu fördern.

Mit diesen fünf Maßnahmen wird sehr aktiv versucht, die Besucher der Landesausstellung erstens für die gesamte Region und nicht bloß für die Ausstellungsstandorte zu interessieren und sie zweitens zu wiederholten Besuchen in der gesamten Region zu motivieren - auch nach dem Ende der Ausstellung. Damit sollen die dezentralen endogenen Potenziale der Region im Bereich Naherholung und Tourismus nachhaltig gestärkt werden, nämlich An-/Abreise mit der Mariazellerbahn, keine räumlichen Konzentrationen von Angeboten/Nachfragern an wenigen Punkten, Gestaltung der Angebote durch lokale und regionale Akteure, Aus-/Fortbildung der Anbieter am Ort auch in Bezug auf das Wissen um die Region sowie nicht das Schaffen künstlicher Erlebniswelten, sondern Aufwertung der vorhandenen dezentralen Potenziale in den Bereichen Natur, Geschichte und Kultur.

Damit werden die Leitideen der eigenständigen Regionalentwicklung aktiv umgesetzt, allerdings in erster Linie im Bereich Naherholung und Tourismus. Dies strahlt zwar auch auf andere Bereiche der Regionalentwicklung aus (in erster Linie auf Landwirtschaft und Regionalprodukte), stellt jedoch nur einen Ausschnitt aus dem breiten Programm der Aufwertungen dar, die durch eine eigenständige Regionalentwicklung initiiert werden sollen.

\subsection{Erste Zwischenergebnisse}

Zum Zeitpunkt des Erstellens dieses Artikels im August 2015 lassen sich bereits erste Zwischenergebnisse festhalten. Eine erste Diskussion um die durch die Landesausstellung ausgelösten 
neuen Möglichkeiten entstand bereits in der Bewerbungsphase um den Ort der Ausstellung im ersten Halbjahr 2012. Eine zweite Diskussionsphase entwickelte sich im Verlauf der Vorbereitungen in den Jahren 2013 und 2014, und hiervon wurden neben der Politik jetzt auch zahlreiche Betriebe (,Regionspartner“) und die Bevölkerung („Naturvermittler“, „ÖTSCHER:REICH-Stationen“) erfasst. Und die Eröffnung der Landesausstellung am 24. April 2015 wurde dann zum Großereignis, das den Alltag der gesamten Ötscherregion sehr stark beeinflusste, genauso wie die Landesausstellung insgesamt sehr öffentlichkeitswirksam überregional vermarktet wurde.

Am 30. Juli 2015 konnte der 100.000. Ausstellungsbesucher begrüßt werden, und bis zum Ende der Ausstellungszeit am 1. November 2015 werden etwa 300.000 Besucher erwartet. Das Ötscherwanderbuch musste bereits kurz nach Erscheinen nachgedruckt werden, der Katalogverkauf läuft gut, und die zusätzlichen Angebote um die Landesausstellung werden lebhaft wahrgenommen. Damit erfüllt diese Landesausstellung die in sie gesetzten Erwartungen voll und ganz, und die dadurch ausgelöste Nachfrage belebt die Ötscherregion auf eine spürbare Weise.

Bei den Menschen der Region wirkten sich die zahlreichen Diskussionen und Vorbereitungen ab 2012 und erst recht die Landesausstellung selbst positiv auf ihr kulturelles Selbstbewusstsein aus. Von den zahlreichen Veranstaltungen, die parallel zur Ausstellung an vielen Orten stattfanden, ist besonders das Theaterprojekt „Die Wahl - ein Projekt zur Stärkung der Zivilgesellschaft in Lunz am See“ zu erwähnen, weil darin zentrale Fragen zur gegenwärtigen Situation und zur Zukunft aufgeworfen wurden; damit wurde eine der Intentionen der Landesausstellung in einem ganz anderen Rahmen mittels eines ganz anderen Mediums aufgegriffen, was als kulturell innovativ bewertet werden kann.

Man kann heute schon feststellen, dass die Landesausstellung in der Region eine Art Aufbruchsstimmung ausgelöst hat, die mit zahlreichen dezentralen Impulsen, Innovationen und Investitionen verbunden ist. Die zentrale Frage ist aber, was davon nach dem Ende der Landesausstellung übrigbleiben wird.

\subsection{Zur zukünftigen Entwicklung}

Eine Ausstellung als ein punktuelles und kurzfristiges Ereignis ist eigentlich nicht dazu geeignet, eine periphere und strukturschwache Region aufzuwerten, weil es dazu flächenhafter und langfristiger Impulse bedarf. Mit der inhaltlichen Gestaltung der Landesausstellung in der Ötscherregion wurde bereits systematisch damit begonnen, das punktuelle in ein flächenhaftes Ereignis umzuwandeln. Die zweite Aufgabe, aus dem einmaligen, zeitlich limitierten Ereignis eine dauerhafte Entwicklung zu machen, fällt dagegen wesentlich schwerer; aber auch hierfür gibt es bereits Ansätze.

Die große Herausforderung besteht darin, auch nach dem Ende der Landesausstellung, dem Abbau der Ausstellungen und dem Versiegen der Besucherströme die Ötscherregion weiterhin für Naherholer und Urlauber attraktiv zu halten. Dies setzt voraus, dass die für die Ausstellung geschaffenen neuen Angebote - Außenstationen, Regionspartner, Naturvermittler, (Rund-)Wanderwege, Ötscher-Basis - weiterhin gepflegt und aktiv vermarktet werden - auch ohne die verbindende Klammer der Landesausstellung. Alle Erfahrungen zeigen, dass dies erstens nicht von den einzelnen Akteuren allein geleistet werden kann, sondern ein gemeinsames, regional koordiniertes Vorgehen erfordert, und dass dies zweitens nur dann gelingt, wenn die Angebote nicht einfach nur perpetuiert, sondern zugleich auch weiterentwickelt und neuen Bedürfnissen angepasst werden.

$\mathrm{Zu}$ diesem Zweck ist der Aufbau einer regionalen Struktur erforderlich, deren Ziel die nachhaltige Entwicklung und Stärkung der gesamten Ötscherregion sein müsste. Da der von der Landesausstellung initiierte Regionszuschnitt quer zu allen politischen Strukturen steht, gibt es bislang eine solche Struktur nicht, und sie müsste erst neu aufgebaut werden. Mitglieder müssten auf jeden Fall alle betroffenen Gemeinden sein sowie weitere Akteure aus Politik, Wirtschaft, Umwelt, Kultur und Zivilgesellschaft, die gemeinsam verbindliche Ziele für die Entwicklung ,,ihrer“ Region erarbeiten. 
Dazu bedarf es einer Art kultureller Aufbruchsstimmung und einer starken regionalen Identität. Als Regionalmanager könnte der Naturpark Ötscher-Tormäuer fungieren, dessen Gebiet bereits grenzüberschreitend angelegt ist und dessen Aufgabe darin besteht, die vier Bereiche „Schutz - Erholung - Bildung - Regionalentwicklung“" auf gleichberechtigte Weise zu fördern und zu entwickeln und daraus eine „Modellregion für Nachhaltige Entwicklung“ zu machen (NATURPARK 2015, S. 6). Weil die Gebietszuschnitte und die Aufgabenstellungen der Naturparke mit den Aufgaben eines Regionalmanagements oft identisch sind, wird den Naturparken derzeit gern die Aufgabe eines Regionalmanagers übertragen (zur Situation in Deutschland vgl. Weber 2013 und Weber \& Weber 2015), und dies könnte auch für die Ötscherregion eine sehr sinnvolle Lösung darstellen (vgl. HeINTEL \& WeIXLBAUMER 2004).

Die erste - und sehr dringliche - Aufgabe dieses Regionalmanagements müsste es sein, dafür zu sorgen, dass die im Rahmen der Landesausstellung entwickelten dezentralen Angebote weitergeführt werden und dass sie - da ja die Landesausstellung im Sommer stattfand - um spezifische Winterangebote ergänzt werden. Weiterhin wäre es sehr wichtig, über die Bereiche Naherholung/ Tourismus hinaus auch Aktivitäten in den Bereichen Landwirtschaft, Gewerbe, kommunale Dienstleistungen (Sechs Gemeinden haben weniger als 610 Einwohner!) und Bildung zu entwickeln sowie die Frage nach der Zukunftsentwicklung (Regionsleitbild) breit zu diskutieren, um die Ötscherregion nachhaltig zu stärken.

Mit dem Aufbau eines solchen Regionalmanagements würde der zeitlich befristete Impuls der Landesausstellung in eine dauerhafte Struktur überführt werden. Die Aktivitäten dazu müssten aber von den Akteuren der Region ausgehen und könnten nicht von der Landesausstellung kommen, die dafür weder kompetent noch zuständig ist - darauf wurde von den Mitarbeitern der Landesausstellung immer wieder hingewiesen. Derzeit gibt es darüber in der Region eine lebhafte Diskussion, und in der ersten Novemberwoche 2015, also unmittelbar nach Ausstellungsende, wird auf einer regionalen Tagung öffentlich gemeinsam diskutiert, wie es weitergehen soll. Dann wird sich zeigen, ob dieses außergewöhnliche Experiment dauerhaft wird oder nicht.

\section{Die theoretischen Hintergründe dieser Landesausstellung}

\subsection{Eigenständige Regionalentwicklung}

„Seine theoriegeschichtlichen Ursprünge hat das Konzept der eigenständigen Regionalentwicklung in der entwicklungspolitischen Diskussion. Auf der Grundlage von Dependenz-, Polarisationsund Zentrum-Peripherie-Theorien wird für ,selektive Abkopplung' bzw. ,Dissoziation ' unterentwickelter Länder vom Weltmarkt plädiert. [...] Dies wurde von der Ebene der Entwicklungsländer auf die Ebene kleinmaßstäblicher Regionen der westlichen Industrieländer übertragen.“ (ERMANN 1998, S. 4)

Ursache für diese Übertragung war das Unbehagen über die räumliche Entwicklung der ländlichen und peripheren Regionen, in denen immer mehr Arbeitsplätze verschwanden, und über die staatliche Politik der Zentralen Orte, die dies noch zusätzlich verstärkte. Mit der eigenständigen oder auch endogenen Regionalentwicklung wurde eine Alternative dazu formuliert, deren Ziel darin bestand, die ländlichen und peripheren Räume als dezentrale Lebens- und Wirtschaftsräume lebendig zu erhalten.

In der zweiten Hälfte der 1970er Jahre gab es in einigen europäischen Staaten Experimente mit der eigenständigen Regionalentwicklung, die aber bald wieder abgebrochen wurden. Nur in Österreich erhielt dieses Konzept eine relevante Bedeutung (Pongratz \& KreIL 1991, S. 91; SAUberer et 
al. 1989; HeINTEL 1994): Es wurde 1979 zur Grundlage der „Sonderaktion für entwicklungsschwache Berggebiete“ des Bundeskanzleramtes, fand 1981 Eingang in das Österreichische Raumordnungskonzept (Glatz \& SCHEER 1981; ÖROK 1981, S. 51 f.) und führte 1983 zum Aufbau der Österreichischen Arbeitsgemeinschaft für eigenständige Regionalentwicklung (ÖAR), die bis heute besteht (allerdings ab 1990 privatisiert). Da innerhalb Österreichs Niederösterreich dasjenige Bundesland war, in dem die eigenständige Regionalentwicklung den größten Stellenwert erlangte (vgl. Abschnitt 4.2), spielte sie auch für die Niederösterreichischen Landesausstellungen eine wichtige Rolle.

Die Leitidee der eigenständigen Regionalentwicklung besteht darin, das Wirtschaften nicht auf wenige große Betriebe zu konzentrieren, die hochspezialisiert für den weltweiten Export produzieren, sondern die zahlreichen dezentralen regionalen Potenziale zu nutzen und damit regionale Wertschöpfungsketten aufzubauen, um die regionale Wertschöpfung zu erhöhen. Dabei wird Wirtschaften nicht als isolierter Prozess gesehen, sondern das regionale Wirtschaften soll so gestaltet werden, dass es umwelt- und sozialverträglich ausgeübt werden kann (Integration von Wirtschaft-Gesellschaft-Umwelt). Schlüsselfaktor ist nicht das Kapital, sondern die regionale Identität, die dazu führt, dass Menschen für ,ihre“ Region Verantwortung übernehmen und innovativ werden, um neue Lebens- und Wirtschaftsperspektiven zu entwickeln. In der konkreten Umsetzung kommt daher „Regionalbetreuern“ (Pongratz \& KREIL 1991, S. 94) eine wichtige Funktion zu, die die regionale Identität stärken und die regionalen Akteure unterstützen und fördern.

Allerdings gibt es für die eigenständige Regionalentwicklung keine allgemein anerkannte Theorie, sondern verschiedene Akteure interpretieren sie auf durchaus unterschiedliche Weise. Die Interpretationsmöglichkeiten reichen von alternativen Autarkievorstellungen bis hin zur Komplementarität mit dem Weltmarkt, und die Identifizierung der nachhaltigen mit der eigenständigen Regionalentwicklung seit den 1990er Jahren erhöht diese Schwierigkeiten noch einmal (ERMANN 1998, S. 8 f.).

Trotz dieser theoretischen Unschärfen sind die drei zentralen Pfeiler der eigenständigen Regionalentwicklung - der zentrale Stellenwert der regionalen Akteure, die große Bedeutung der dezentralen endogenen Potenziale und die „Integration“ von Wirtschaft, Gesellschaft und Umwelt - allgemein anerkannt und sie unterscheiden sich signifikant von anderen Konzepten der Regionalentwicklung.

\subsection{Die niederösterreichische Regionalpolitik}

Angesichts der Tatsache, dass in Niederösterreich innovative regionalpolitische Ansätze entworfen und umgesetzt wurden, kann man im Bereich der Regionalpolitik von einem „Modell Niederösterreich“ sprechen (SCHWARz 2008, S. 51).

Wie auch in anderen Bundesländern beginnt Raumordnung und Regionalpolitik hier 1972 als „,zentralistisch-technokratische Planung und Territorialentwicklung ,von oben“ “ mittels des Systems Zentraler Orte und sektoraler Raumordnungsprogramme (Schwarz 2008, S. 55). Da sich damit aber die räumlichen Disparitäten noch weiter verschärften, statt geringer zu werden (ScHwARz 2008, S. 55), gab es 1982 einen radikalen Kurswechsel hin zur eigenständigen Regionalentwicklung, wobei das Land Niederösterreich innerhalb Österreichs „Zweifelsohne eine Vorreiterrolle“ spielte (Schwarz 2008, S. 59). Dabei stellte sich aber schnell heraus, dass regionale und lokale Akteure trotz ihrer großen theoretischen Bedeutung immer wieder in der Gefahr standen, bornierte Interessen (Gemeindeegoismen, Kirchturmdenken, private Hobbies) zu vertreten (HeINTEL 1994).

Deshalb entwickelte das Land Niederösterreich einen eigenen Weg, indem es die Vorteile der kreativen Entwicklungsansätze (bottom-up) mit den traditionellen Stärken der zentralen Förderstellen (top-down) zu einer „Regionalpolitik des mittleren Weges“ verband (SchwARz 2008, S. 62).

Zwar gab es anschließend weitere Paradigmenwechsel in der Regionalpolitik (1988: Innovationsorientierte Regionalpolitik; 1995: EU-Regionalpolitik; 2007: Lissabon-Strategie der EU auf regionaler Ebene; ScHWARz 2008, S. 62-69), aber es lässt sich feststellen, dass dabei zentrale Ele- 
mente der eigenständigen Regionalentwicklung bis heute eine wichtige Rolle spielen. Dies zeigt sich u.a. daran, dass als Reaktion auf die Lissabon-Strategie der EU das Land Niederösterreich den Stellenwert der Region als Gegengewicht gegen die negativen Folgen der Globalisierung weiter stärkt (SCHWARZ 2008, S. 68).

Die konkrete Regionalpolitik ist seit dem Landesentwicklungskonzept von 2004 (АMT DER NIEDERÖSTERREICHISCHEN LANDESREGIERUNG 2004) dadurch geprägt, dass regionale Leitbilder für die fünf „Hauptregionen“ (Neben den vier traditionellen „Vierteln“ wurde die Region „NÖ-Mitte“ als fünfte Hauptregion ausgewiesen.) entwickelt wurden (AMT DER NIEDERÖSTERREICHISCHEN LANDESREGIERUNG 2005), dass sich unterhalb dieser Ebene „Kleinregionen“ auf dem Prinzip der Freiwilligkeit in Form gemeindeübergreifender Zusammenarbeit konstituiert haben, von denen es derzeit 58 mit $85 \%$ aller Gemeinden gibt (KRZ 2015), und dass die Dorf- und Stadterneuerung (kommunale Ebene) nach wie vor in Niederösterreich eine wichtige Rolle bei der Aufwertung ländlicher Räume spielt.

Stellt man die Ötscherregion, so wie sie im Rahmen der Landesausstellung 2015 konstituiert und abgegrenzt wurde, in den Kontext der niederösterreichischen Raumordnung, so steht sie quer zu allen bestehenden Raumstrukturen: Sie liegt zur Hälfte in der Hauptregion Mostviertel und zur Hälfte in der Hauptregion NÖ-Mitte (AMT DER NIEDERÖSTERREICHISCHEN LANDESREGIERUNG 2005, S. 27), und auch die Kleinregionen sind nicht einmal ansatzweise mit der Ötscherregion identisch (vgl. die Karten „Kleinregionen im Mostviertel“ und „Kleinregionen NÖ-Mitte“ in KRZ 2015).

Seit 2013 wird der bemerkenswerte und innovative Versuch gemacht, die vier Ebenen der Raumordnung (Land, Hauptregionen, Kleinregionen, Kommunen) besser miteinander zu vernetzen und die Fördermaßnahmen gezielter zu bündeln, wozu eine neue Struktur, die „NÖ.Regional GmbH“ gegründet wurde, die partizipativ strukturiert ist (ÖROK 2015, S. 180).

\subsection{Die Niederösterreichischen Landesausstellungen 1960-2015}

Das ursprüngliche Ziel der Niederösterreichischen Landesausstellungen bestand darin, ,zur Festigung eines Landesbewusstseins“ beizutragen, das im Jahr 1960 „durchaus unterentwickelt“ (Peter KAMPITS in Fritz \& Linke 2011, S. 175) oder sogar „schwer beschädigt“ war (Thomas JORDA in FRITZ \& LiNKE 2011, S. 178). Zu diesem Zweck wurden (kunst-)geschichtliche Themen mit starkem Niederösterreichbezug gewählt (Biedermeier, Friedrich III., Römer an der Donau, Renaissance, Babenberger, frühe Habsburger, Kaiser Joseph II. usw.), und die Ausstellungen fanden in bedeutenden denkmalgeschützten Objekten (oft Burgen und Schlössern) statt, die dadurch einem breiten Publikum - auch über Niederösterreich hinaus - bekannt gemacht werden sollten. Durch umfangreiche Restaurierungen der denkmalgeschützten Gebäude, gezielte Infrastrukturverbesserungen in den Ausstellungsorten und die Verbesserung ihrer Erreichbarkeit profitierte schon damals die regionale Wirtschaft von den Landesausstellungen (Erwin PrölL in FrITZ \& LinKe 2011, S. 7).

Am Ende der 1980er Jahre wandte man sich vor dem Hintergrund einer inzwischen stabilen niederösterreichischen Identität von diesen Themen ab und widmete sich breiteren und aktuelleren Themen wie „Kunst des Heilens“, „Frauenleben in Österreich“, „Sein \& Sinn, Burg \& Mensch“, „Theaterwelt - Welttheater“, wobei jetzt aber die Tendenz hin zu einer gewissen inhaltlichen Beliebigkeit entstand (Thomas JoRDA in Fritz \& LinKe 2011, S. 179). Parallel dazu änderte sich auch das Publikumsinteresse, und an die frühere Stelle „höchsten wissenschaftlichen Anspruch““ (a.a.O., S. 178) trat jetzt das Bedürfnis, die Inhalte auf ,leicht fassbare, unterhaltsame“ Weise zu präsentieren (a.a.O., S. 179). Zugleich wurde das Rahmenprogramm der Landesausstellungen vor dem Hintergrund eines veränderten Freizeitverhaltens immer wichtiger, sodass Thomas JORDA die damaligen Landesausstellungen als ,eine Art Gesamtkunstwerk“ bezeichnet (a.a.O., S. 179).

Im Jahr 2007 setzte eine neue Entwicklung ein, indem erstmals mit „Feuer \& Erde“ eine Region die Niederösterreichische Eisenstraße - in das Zentrum einer Landesausstellung trat, und diese Aus- 
richtung auf eine Region wurde seither beibehalten. Allerdings wurde der Bezug zur Ausstellungsregion in den Jahren 2007 bis 2011 durch externe Experten, also top-down, umgesetzt, weshalb das Ende der Ausstellung jeweils auch das Ende der regionalen Aktivitäten bedeutete. In einem zweiten Schritt wurde deshalb für die Landesausstellung 2013 eine breite Bürgerbeteiligung initiiert, aber erst mit der Landesausstellung 2015 wurden auch dezentrale Angebote aus der Region selbst entwickelt, wurde also die neue Ausrichtung weiter perfektioniert (mündliche Mitteilung Kurt FARASIN).

Die neue Leitidee der Landesausstellungen kann man so beschreiben: Im Zeitalter der Globalisierung liegt der Fokus ganz bewusst auf einer Region (nicht verstanden als isolierte Insel, sondern einschließlich ihrer übergeordneten Verflechtungen), die mit ihrer spezifischen Natur, Geschichte, Wirtschaft und Kultur ein Gegengewicht zur austauschbaren globalisierten Welt bildet. Für die Besucher der Ausstellung steht das Wahrnehmen, Verstehen und Erleben dieser regionalen Spezifika - sowohl konzentriert am Ausstellungsort als auch verteilt in der gesamten Region - im Zentrum, für die Region geht es zentral um die Auseinandersetzung mit ihrer eigenen Situation, um die Aufwertung ihrer endogenen dezentralen Potenziale und um ihre zukünftige Entwicklung, wobei sich beide Standpunkte - Regionsbesucher und Regionsbewohner - wechselseitig bereichern.

\section{Bilanz: Eine Landesausstellung als Initiator einer eigenständigen und nachhal- tigen Regionalentwicklung}

Bei dieser Landesausstellung handelt es sich aus zwei Gründen um ein außergewöhnliches Projekt:

1. Eine räumlich und zeitlich eng begrenzte Ausstellung ist eigentlich kein geeigneter Rahmen, um eine Region nachhaltig, also flächenhaft und langfristig aufzuwerten. Die Erfahrungen mit der Niederösterreichischen Landesausstellung 2015 zeigen aber, unter welchen Voraussetzungen und Rahmenbedingungen dies trotzdem möglich ist. Allerdings kann eine solche Ausstellung dafür nur einen ersten - aber durchaus entscheidenden - Impuls geben; anschließend muss die Region die weitere Entwicklung in die eigenen Hände nehmen.

2. Vor dem Hintergrund des verbreiteten neoliberalen Denkens würde die Aufwertung einer peripheren Region normalerweise über die Verbesserung der Erreichbarkeit und ein touristisches Großprojekt realisiert werden. Die Landesausstellung geht mit der Aufwertung der dezentralen Ressourcen und Potenziale dagegen einen ganz anderen, heute ungewöhnlichen Weg, und sie zeigt, dass dieser Weg durchaus erfolgversprechend sein kann.

Beide Gründe zusammen machen aus diesem außergewöhnlichen ein innovatives Projekt, weil es - quer zu allen bestehenden Strukturen und Selbstverständlichkeiten - Dinge miteinander verbindet, die üblicherweise als nicht vereinbar angesehen werden. Dadurch eröffnet diese Landesausstellung neue Wege und umgeht alte Blockaden.

Diese Innovation wäre wohl nicht möglich gewesen, wenn nicht in Niederösterreich die eigenständige Regionalentwicklung seit 1982 einen wichtigen Stellenwert gehabt hätte, sodass die aktuellen Herausforderungen der Globalisierung hier zur Aufwertung und nicht zur Abwertung der Region geführt haben. Die Suche nach neuen Themen und Aufgaben für die Niederösterreichischen Landesausstellungen wurde dadurch mitgeprägt, und ihre Umsetzung konnte von den jahrzehntelangen Erfahrungen in diesem Bereich profitieren.

Nachdem jetzt dieses völlig neue Modell für eine Landesausstellung vorliegt und seine Bewährungsprobe bestanden hat, bietet es sich zur Nachahmung auch in anderen Regionen an: Überall in den Alpen und in Europa finden regelmäßig Ausstellungen auf Staats-, Länder- oder Regionsebene statt, die immer nur punktuelle Ereignisse sind. Diese Ausstellungen auf die gesamte Ausstellungsregion hin zu öffnen, dürfte wohl für alle Betroffenen - Veranstalter, Akteure, Besucher - interessant 
sein, selbst in Fällen, in denen Ausstellungsbudget und Besucherzahlen wesentlich kleiner sind als in Niederösterreich. Und häufig sucht man dabei nach geeigneten, nicht austauschbaren Themen, sodass es sich anbietet, die eigene Region mit ihren Spezifika zum Gegenstand zu machen.

Es bleibt zu hoffen, dass dieses innovative Projekt in möglichst vielen anderen Regionen wiederholt und dabei weiterentwickelt wird und dass dadurch neue Wege und Möglichkeiten zur dezentralen Aufwertung ländlicher Räume im Zeitalter der Globalisierung eröffnet werden.

\section{Literaturverzeichnis}

Amt der NiederösterReichischen LandeSRegierung (Hrsg.) (2004), Strategie Niederösterreich. Landesentwicklungskonzept für Niederösterreich. Prinzipien, Grundsätze und Ziele einer integrierten Raumentwicklung. St. Pölten.

Amt Der Niederösterreichischen Landesregierung (Hrsg.) (2005), Perspektiven für die Hauptregionen. Waldviertel, Weinviertel, NÖ-Mitte, Industrieviertel, Mostviertel. St. Pölten.

BätzıNG W. (2015a), Die Alpen. Geschichte und Zukunft einer europäischen Kulturlandschaft. München, vollständig überarbeitete und erweiterte Auflage.

BätzıNG W. (2015b), Zwischen Wildnis und Freizeitpark. Eine Streitschrift zur Zukunft der Alpen. Zürich, Rotpunktverlag.

BätzıNG W. (2015c), Der Ötscher und die Alpen. Alpentypische Entwicklungen und regionale Besonderheiten. In: Schallaburg Kulturbetriebsges.m.B.H. (Hrsg.), ÖTSCHER:REICH. Die Alpen und wir. Katalog zur Niederösterreichischen Landesausstellung 2015. Schallaburg, S. $110-141$ und 186-223.

BätZING W. (2015d), Gegenwart und Zukunft der Ötscherregion im alpenweiten Vergleich. In: BruCKMÜLLER E. (Hrsg.), Im Reich des Ötschers. Zur Vielfalt einer Region, S. 22-37. Wien, new academic press.

BätzıNG W. (2015e), Die Ötscherregion - ein neuer Blick auf die Alpen. Vortrag anlässlich der Eröffnung der Niederösterreichischen Landesausstellung 2015 am 24. April 2015 in Laubenbachmühle. Video-Mitschnitt: www.youtube.com/watch? $v=j N S b$ VaudW18.

Bätzing W., HofFert-Hösl H. (2015), Der Ötscher. Wanderungen in den Niederösterreichischen Kalkalpen. Zürich, Rotpunktverlag.

BÄtzING W. (2014), Die territoriale Neugliederung Bayerns durch die Gebietsreform der 1970er Jahre als Antwort auf die Herausforderungen der Moderne. Eine grundsätzliche Bewertung dieser säkularen Reform und ihre Bedeutung für die heutigen Herausforderungen am Beispiel des Landkreises Neumarkt in der Oberpfalz. In: Mitteilungen der Fränkischen Geographischen Gesellschaft, 59, S. 151-164.

Boesch M. (1989), Engagierte Geographie. Zur Rekonstruktion der Raumwissenschaft als politikorientierte Geographie (= Erdkundliches Wissen, 98). Stuttgart, Franz Steiner Verlag.

Borsdorf A. (Hrsg.) (2005), Das neue Bild Österreichs. Strukturen und Entwicklungen im Alpenraum und in den Vorländern. Wien, Verlag der Österreichischen Akademie der Wissenschaften.

BrucKMÜLLER E. (Hrsg.) (2015), Im Reich des Ötschers. Zur Vielfalt einer Region (= Austriaca, Schriftenreihe des Instituts für Österreichkunde). Wien, new academic press.

ERMANN U. (1998), Regionale Wirtschaftsverflechtungen fränkischer Brauereien. Perspektiven für eine eigenständige und nachhaltige Regionalentwicklung (= Erlanger Geographische Arbeiten, Sonderband 25). Erlangen, Palm und Enke.

Fritz P., Linke R. (Red.) (2011), 50 Jahre Landesausstellungen Niederösterreich. St. Pölten, Amt der Niederösterreichischen Landesregierung/Abteilung Kunst und Kultur. 
Glatz H., SCHEER G. (1981), Eigenständige Regionalentwicklung - ein Weg für strukturell benachteiligte Gebiete in Österreich (= Raumplanung in Österreich, 1). Wien, Bundeskanzleramt/ Bundespressedienst.

Heintel M. (1994), Endogene Regionalentwicklung. Eine wirtschaftspolitische Alternative für struktur- und entwicklungsschwache Regionen? (= Mitteilungen des Arbeitskreises für Regionalforschung, Sonderband 5). Wien, Arbeitskreis Regionalforschung.

Heintel M., Weixlbaumer N. (2004), Gebietsschutz und Regionalmanagement. Erfahrungen und Empfehlungen anhand des Naturparks Ötscher-Tormäuer. In: Geographischer Jahresbericht aus Österreich, LX/LXI, S. 149-174.

KleinRegionale ZusammenarbeIt (KRZ) (2015), www.noe.gv.at/Land-Zukunft/Raumordnung/Kleinregionen.html (letzter Zugriff 17.07.2015).

Krebs N. (1928), Die Ostalpen und das heutige Österreich. Eine Länderkunde. Zweiter Band: Regionaler Teil. Darmstadt 1961, Wissenschaftliche Buchgesellschaft. (unveränderter Nachdruck der Ausgabe Stuttgart 1928).

NAtURPARK (Hrsg.) (2015), Positionspapier Naturpark Ötscher-Tormäuer vom 5. Februar 2015. Wieselburg.

ÖSTERREICHISCHE RAUMORDNUNGSKONFERENZ (ÖROK) (Hrsg.) (2015), 14. Raumordnungsbericht. Analysen und Berichte zur räumlichen Entwicklung Österreichs 2012-2014 (= ÖROK-Schriftenreihe, 195). Wien.

ÖSTERREICHISCHE RAUMORDNUNGSKONFERENZ (ÖROK) (Hrsg.) (1981), Österreichisches Raumordnungskonzept (= ÖROK-Schriftenreihe, 28). Wien.

Pongratz H., KreIL M. (1991), Möglichkeiten einer eigenständigen Regionalentwicklung. In: Zeitschrift für Agrargeschichte und Agrarsoziologie, 39, 1, S. 91-111.

Schallaburg (Hrsg.) (2015), ÖTSCHER:REICH. Die Alpen und wir. Katalog zur Niederösterreichischen Landesausstellung 2015. Schallaburg.

Sauberer M., Schindegger F., TöDtling F. (1989), Strategien für entwicklungsschwache Problemgebiete (= ÖROK-Schriftenreihe, 77). Wien, ÖROK.

Schwarz W. (2008), Wege und Perspektiven der Regionalpolitik in Niederösterreich - mehrfacher thematisch-strategischer Paradigmenwechsel. In: Mitteilungen der Österreichischen Geographischen Gesellschaft, 150, S. 49-74.

Sondershaus F. (2008), Eigenständige Regionalentwicklung und nachhaltige Entwicklungsräume. Überlegungen zum Raumbezug eigenständiger Entwicklungskonzepte vor dem Beispiel des administrativ geteilten Raumes Fränkische Schweiz. In: Mitteilungen der Fränkischen Geographischen Gesellschaft, 55, S. 15-47.

Weber F. (2013), Naturparke als Manager einer nachhaltigen Regionalentwicklung. Probleme, Potenziale und Lösungsansätze. Wiesbaden, Springer VS.

Weber Fr., Weber Fl. (2015), Naturparke und die Aufgabe der nachhaltigen Regionalentwicklung. Jenseits von Wanderwegemarkierern und Parkbankaufstellern. In: Naturschutz und Landschaftsplanung, 47, 5, S. 149-156.

\section{Internetseiten:}

NIEDERÖSTERREICHISCHE LANDESAUSSTELLUNG: www.noe-landesausstellung.at

SCHALlABURG KULtURBETRIEBSGES.м.в.H.: www.noeku.at/de/gesellschaften/ausstellungsbetriebe/ schallaburg

RAUMORDNUNG IN NIEDERÖSTERREICH: www.noe.gv.at/Land-Zukunft/Raumordnung.html

ÖAR: www.oear.at

NATURPARK ÖTSCHER-TORMÄUER: www.naturpark-oetscher.at 
MARIAZELLERBAHN: www.noevog.at/mariazellerbahn

WILDNISGEBIET DÜRRENSTEIN: www.wildnisgebiet.at

MostViertel TOuRISMus GmbH: www.mostviertel.at

EISENSTRASSE NIEDERÖSTERREICH: $w$ ww.eisenstrasse.at

MOSTVIERTLER NACHHALTIGKEITSKONFERENZEN: www.mostviertel.at/nachhaltigkeitskonferenz

THEATERPROJEKT „DIE WAHL“: www.wellenklaenge.at 